\title{
FAUNA EDÁFICA EM ÁREA PERIODICAMENTE INUNDÁVEL NA RESTINGA DA MARAMBAIA, RJ ${ }^{(1)}$
}

\author{
Cristiane Figueira da Silva ${ }^{(2)}$, Guilherme Henrique Almeida Pereira ${ }^{(3)}$, Marcos Gervasio \\ Pereira $^{(4)}$, Avelino Nogueira da Silva ${ }^{(5)}$ \& Luis Fernando Tavares de Menezes ${ }^{(6)}$
}

\begin{abstract}
RESUMO
No compartimento serapilheira-solo, a fauna do solo exerce importante papel na disponibilização de nutrientes, uma vez que é responsável pela regulação da comunidade de microrganismos decompositores da matéria orgânica e pela fragmentação desse material. Este trabalho objetivou avaliar a riqueza e abundância da fauna edáfica, bem como sua distribuição vertical, em área de restinga periodicamente inundada na Ilha da Marambaia, RJ, em duas épocas distintas, verão (chuvosa) e inverno (seca). Foram coletadas 10 amostras de serapilheira e de solo na profundidade de $0-5 \mathrm{~cm}$, em que foram feitas extrações da fauna por meio de extratores do tipo Berlese-Tüllgren. Observou-se aumento da abundância da fauna edáfica do período seco para o chuvoso, enquanto a riqueza de grupos taxonômicos não foi modificada. Em ambas as épocas, os grupos de maior ocorrência foram Formicidae e Collembola. Quanto à distribuição vertical, verificou-se a preferência dos organismos pelo compartimento serapilheira, no período chuvoso, e pelo solo, no período seco. A microfagia e a saprofagia representaram as principais formas de utilização dos recursos alimentares, seguidos da predação, sugerindo melhor estruturação do habitat no ecossistema. Além disso, a ocorrência de grupos que só são percebidos em ambientes não perturbados indica que o ecossistema de restinga da Ilha da Marambaia, no momento em que foi avaliado, ano de 2002, encontrava-se pouco ou nada degradado e em bom estado de conservação.
\end{abstract}

Termos de indexação: serapilheira, solo, invertebrados.

(1) Projeto Apoiado pela Fundação Boticário Proteção a Natureza e pela FAPERJ. Recebido para publicação em 19 de novembro de 2012 e aprovado em 12 de abril de 2013.

(2) Pós-Doutoranda do Curso de Pós-Graduação em Agronomia - Ciência do Solo, UFRRJ. BR 465, km 7. CEP 23890-000 Seropédica (RJ). Bolsista da CAPES. E-mail: cfigueirasilva@yahoo.com.br

(3) Mestrando do Programa de Pós-Graduação em Ciências Ambientais e Florestais, UFRRJ. Bolsista da CAPES. E-mail: guilhermepereira06@gmail.com

(4) Professor Associado IV da Universidade Federal Rural do Rio de Janeiro (UFRRJ). Bolsista do CNPq e Cientista do Nosso Estado da FAPERJ. E-mail:gervasio@ufrrj.br

(5) Mestre em Sensoriamente Remoto, Grupo de Perícias em Meio Ambiente do Setor Técnico-Científico do Departamento de Polícia Federal Superintendência Regional do Rio de Janeiro, Av. Rodrigues Alves, 1, Bairro Centro. CEP 20081-250 Rio de Janeiro (RJ). E-mail: avelinoufrrj@hotmail.com

(6) Professor Adjunto III da Universidade Federal do Espírito Santo, Centro Universitário Norte do Espírito Santo. BR 101 Norte, km 60, Litorâneo. CEP 29932-540 São Mateus (ES). E-mail: ltmenezes@gmail.com 


\title{
SUMMARY: SOIL FAUNA IN A PERIODICALLY FLOODED RESTINGA SALT MARSH AREA IN MARAMBAIA, RJ
}

\begin{abstract}
In the litter-soil compartment, soil fauna plays an important role in nutrient availability for plants, since it regulates the microbial community involved with organic matter decomposition and fragmentation. The aim of this study was to evaluate the richness and abundance of soil fauna as well as its vertical distribution on a periodically flooded sandbank area ("restinga") in Marambaia Island - RJ, in two season (summer -rainy and winter-dry). Litter and soil was sampled $(0-5 \mathrm{~cm})$, from which fauna was extracted by Berlese-Tüllgren extractors. An increase in the abundance of soil fauna was observed from the dry to the rainy season, while the abundance of taxonomic groups was not modified. In both seasons, the most frequent groups were Formicidae and Collembola. Regarding the vertical distribution, the soil fauna preferred litter in the rainy and soil in the dry period. The microphagy and saprophagy represented the main forms of resource use, followed by predation, suggesting a sound structure of the habitat in the ecosystem. Moreover, the occurrence of groups that are found in undisturbed environments only, indicated that the ecosystem Restinga on the Island of Marambaia, at the time of evaluation (2002), was little or not degraded and well-conserved.
\end{abstract}

Index terms: litter, soil, invertebrates.

\section{INTRODUÇÃO}

Solos sob vegetação de restinga são arenosos e, em geral, apresentam baixos teores de matéria orgânica e limitada capacidade de suporte para o desenvolvimento das plantas. A baixa capacidade de reter água e nutrientes, que são essenciais à manutenção dos componentes biológicos do sistema, faz com que as formas orgânicas e a biota exerçam papel crucial na fertilidade desses solos (Manlay et al., 2000; Gomes et al., 2010). Paula et al. (2009) e Pereira et al. (2012) destacam a serapilheira como de grande importância química nesse ambiente, uma vez que a decomposição dela é uma das principais fontes de nutrientes.

A decomposição é um processo essencialmente biológico, em que os organismos, invertebrados e microrganismos, são os principais agentes da desestruturação física e química, que ocorre após a senescência de partes ou até de indivíduos inteiros. Nos ecossistemas terrestres, os invertebrados, também chamados de fauna do solo ou fauna edáfica, podem estar associados ao processo de decomposição no compartimento serapilheira-solo (Lavelle et al., 1993; Yang \& Chen, 2009), em razão da existência de grande diversidade de recursos e dos micro-habitats nesse compartimento (Silva, 1998).

A fauna do solo exerce importante papel na ciclagem de nutrientes (Osler \& Sommerkorn, 2007; Dupont et al., 2009; Carrillo et al., 2011), uma vez que é responsável pela regulação da comunidade de microrganismos decompositores da matéria orgânica e fragmentação desse material (Souza et al., 2008; Yang \& Chen, 2009), influenciando direta e indiretamente no ciclo da matéria e no fluxo de energia ao longo dos ecossistemas terrestres. Além disso, esses organismos apresentam grande contribuição para a agregação do solo (Scheu, 2003; Oyedele et al., 2006;
Oliveira et al., 2012; Siddiky et al., 2012), por meio de suas ações mecânicas (construção de ninhos e escavação) e modificando os materiais do solo pela deposição de compostos orgânicos (Bignell, 2006).

Diferentes fatores podem influenciar a distribuição, composição, abundância e atividade alimentar da fauna do solo (Eggleton et al., 2009; Gomes et al., 2010; Briar et al., 2011; Holmstrup et al., 2012; Simpson et al., 2012). Simpson et al. (2012) enfatizam os possíveis efeitos das mudanças climáticas sobre a atividade da fauna edáfica, principalmente ao que se refere à influência na umidade do solo (Holmstrup et al., 2012). Oscilações de temperatura e umidade e características das estações do ano são fatores que influenciam a densidade faunística (Soares \& Costa, 2001; Fernandes et al., 2011). Alguns autores ressaltam a sensibilidade de grupos taxonômicos da fauna a períodos secos (Fernandes et al., 2011), enquanto outros apresentam estratégias de sobrevivência de organismos, que migram para ambientes (compartimentos soloserapilheira) mais favoráveis (Corrêa Neto et al., 2001; Moço et al., 2005).

Embora seja muito importante no processo de ciclagem de nutrientes, a dinâmica da fauna edáfica em áreas de restinga ainda é pouco conhecida. A determinação da sua população e diversidade é de fundamental importância para avaliar as interações biológicas no sistema solo/planta (Giracca et al., 2003), elucidar os processos de ciclagem de nutrientes e, dessa forma, proporcionar subsídios para a compreensão do funcionamento desses ecossistemas costeiros.

Este trabalho teve como objetivo avaliar a riqueza e abundância dos grupos taxonômicos e funcionais da fauna edáfica no sistema solo-serapilheira, bem como sua distribuição vertical, em área de restinga periodicamente inundada na Ilha da Marambaia, RJ, em duas épocas do ano, chuvosa e seca. 


\section{MATERIAL E MÉTODOS}

A restinga da Marambaia, com aproximadamente $49,40 \mathrm{~km}^{2}$, localiza-se no Estado do Rio de Janeiro, fazendo parte dos municípios do Rio de Janeiro, Itaguaí e Mangaratiba. O clima da região enquadra-se no macroclima Aw - tropical chuvoso, segundo Köppen (1948). As temperaturas do ar são típicas das áreas litorâneas tropicais, com médias mensais acima de $20,0{ }^{\circ} \mathrm{C}$ e a média anual de $23,7^{\circ} \mathrm{C}$. Em fevereiro, ocorre a maior temperatura média mensal $\left(26,8^{\circ} \mathrm{C}\right)$ e, em agosto, a menor, cerca de $20^{\circ} \mathrm{C}$. A precipitação pluvial média anual é de $1.239,7 \mathrm{~mm}$, com $37 \%$ de ocorrência no verão, durante 40 dias chuvosos. Entre novembro e março, os índices pluviométricos médios são sempre superiores a $100 \mathrm{~mm}$. Os meses de inverno são os mais secos, em que ocorrem apenas $15 \%$ da precipitação pluvial média anual, distribuída em cerca de 20 dias; em julho e agosto, situa-se entre 40 e 55 $\mathrm{mm}$ (Mattos, 2005).

A floresta de duna está restrita à extremidade leste da Marambaia, sendo o trecho estudado localizado nas coordenadas $23^{\circ} 03^{\prime} \mathrm{S}$ e $43^{\circ} 36^{\prime} \mathrm{W}$, distando-se aproximadamente $500 \mathrm{~m}$ do oceano. A área de estudo compreende a formação vegetal localizada na praia da Armação, na porção oeste da restinga de Marambaia. Essa formação distingue-se das demais pelo seu porte, em que os indivíduos emergentes podem alcançar $25 \mathrm{~m}$ de altura. O solo é classificado como Neossolo Quartzarênico (Menezes et al., 2005). Por estar inserida em depressões entre cordões arenosos, essa área está sujeita à inundação temporária, em razão do transbordamento do lençol freático. No período de maior pluviosidade, a área permanece encharcada, enquanto nos meses mais secos, há presença de lâminas de água mais espaçadas, localizadas nos pontos mais baixos do relevo (Paula, 2009). A condição de elevada saturação hídrica do solo favorece o acúmulo de espessa camada de material orgânico na superfície dele, que é estimada como sendo superior a $40 \mathrm{~cm}$ (Souza et al., 2008). De acordo com Paula (2009), o teor de carbono orgânico total, nessa área, na camada de 0-20 cm, é em torno de $330 \mathrm{~g} \mathrm{~kg}^{-1}$.

Para a coleta das amostras, foi delimitada uma área de aproximadamente $1 \mathrm{ha}$; realizou-se a amostragem com o auxílio de uma sonda quadrada metálica de $25 \mathrm{~cm}$ de lado, sendo tomadas 10 amostras distribuídas aleatoriamente. Cada amostra foi decomposta em subamostras de serapilheira e de solo superficial, amostrando-se apenas os cinco primeiros centímetros de material de solo. Foram realizadas duas coletas, uma na época úmida (verão) e a outra na seca (inverno), ambas no ano de 2002.

O material coletado foi acondicionado em extratores Berlese-Tüllgren (Garay, 1989) modificado. Na base de cada extrator, foram depositados frascos contendo solução fixadora - solução saturada de ácido acetilsalicilico. Como fontes geradoras de luz e calor, foram utilizadas lâmpadas incandescentes de $40 \mathrm{~W}$, que permaneceram acesas ininterruptamente por 15 dias. Após a extração, as amostras foram triadas com auxílio de lupa binocular e os organismos separados em grandes grupos taxonômicos, por meio da comparação dos indivíduos com pranchas de identificação. Visando à elaboração de um estudo mais refinado (Oliveira, 1997), as larvas de insetos holometábolos foram separadas dos indivíduos adultos, pois, apesar de pertencerem ao mesmo grupo taxonômico, os nichos de ambos diferem fortemente (Correia, 1994). De acordo com Odum (1988), larvas e adultos de uma mesma espécie podem desempenhar papéis ecológicos diferentes.

Os valores obtidos a partir da contagem dos indivíduos foram transformados para indivíduos por $\mathrm{m}^{2}$, tendo sido calculados as médias e os respectivos erros-padrão para cada época. Para verificar se houve diferenças entre as estações do ano, foi usado o teste $\mathrm{T}$ de Wilcoxon.

Com a finalidade de se verificar se houve algum tipo de preferência quanto à distribuição vertical, ou seja, entre a serapilheira e o solo, foi aplicado o teste do Qui-quadrado $\left(\chi^{2}\right)$ (Pimentel-Gomes, 2000). A hipótese nula é a inexistência de qualquer tipo de aderência, ou seja, uma distribuição observada de dados, que se ajusta a uma distribuição esperada sem diferenças entre a serapilheira e o solo, em que corresponde a equação: $\chi^{2}=\Sigma(f o-f e)^{2} / f e$, em que $f o$ corresponde à frequência observada $\mathrm{e} e$ à frequência esperada (Pimentel-Gomes, 2000).

A análise de correspondência canônica foi realizada por meio do programa PAST (Hammer et al., 2004)

\section{RESULTADOS E DISCUSSÃO}

Os resultados da abundância e riqueza dos grupos taxonômicos da fauna do solo e da serapilheira, nas estações chuvosa (verão) e seca (inverno), encontramse no quadro 1 . Não foram verificadas diferenças significativas nas riquezas de grupos taxonômicos em razão da sazonalidade. Dentre os grupos observados, Scorpionida, Lepidoptera e Tricoptera foram os que ocorreram apenas na estação chuvosa, enquanto Dermaptera, Isoptera, Protura e larvas de Hymenoptera, apenas na estação seca (Quadro 1), corroborando com Oliveira (1997) e Fernandes et al. (2011), que, segundo esses autores, muitos grupos da fauna edáfica são influenciados pelas variações ambientais. Nesse estudo, o encharcamento do solo (afloramento do lençol freático) no período de maior pluviosidade (verão), pode ser explicação para a ausência de determinados grupos nessa época.

Com relação à abundância dos artrópodos no sistema solo-serapilheira, encontraram-se os valores de 2.275,2 indivíduos por $\mathrm{m}^{2}$ no inverno e $3.246,4$ indivíduos por $\mathrm{m}^{2}$ no verão, indicando que, do inverno para o verão, a população aumentou. Esse padrão pode ser por causa das temperaturas mais elevadas no 
Quadro 1. Frequência relativa e absoluta da fauna edáfica nos compartimentos solo e serapilheira, nas estações verão e inverno, na restinga da Marambaia, $\mathbf{R J}$

\begin{tabular}{|c|c|c|c|c|c|c|}
\hline \multirow{2}{*}{ Grupo taxonômico } & \multicolumn{3}{|c|}{ Verão } & \multicolumn{3}{|c|}{ Inverno } \\
\hline & \multirow{2}{*}{$\begin{array}{c}\text { Total } \\
\%\end{array}$} & Solo & Serapilheira & \multirow{2}{*}{$\begin{array}{c}\text { Total } \\
\%\end{array}$} & Solo & Serapilheira \\
\hline & & \multicolumn{2}{|c|}{ ind $/ \mathrm{m}^{2} \pm \mathrm{e}^{(1)}$} & & \multicolumn{2}{|c|}{ ind $/ \mathrm{m}^{2} \pm \mathrm{e}^{(1)}$} \\
\hline Formicidae & 28,44 & $505,6 \pm 150,6$ & $417,6 \pm 119,7$ & 28,83 & $470,4 \pm 90,9$ & $185,6 \pm 70,2$ \\
\hline Isoptera & 1,72 & $28,8 \pm 4,9$ & $27,2 \pm 9,8$ & 2,74 & $60,8 \pm 29,9$ & $1,6 \pm 1,6$ \\
\hline Blattodea & 0,05 & - & $1,6 \pm 1,6$ & 0,98 & $8,0 \pm 8,0$ & $14,4 \pm 4,4$ \\
\hline Diplopoda & 0,30 & $9,6 \pm 4,3$ & - & 0,07 & $1,6 \pm 1,6$ & - \\
\hline Diplura & 3,01 & - & $97,6 \pm 32,1$ & 3,16 & $22,4 \pm 5,4$ & $49,6 \pm 16,6$ \\
\hline Psocoptera & 0,34 & $1,6 \pm 1,6$ & $9,6 \pm 4,9$ & 0,35 & $8,0 \pm 4,9$ & - \\
\hline Symphyla & 1,77 & $57,6 \pm 21,0$ & - & 2,46 & $54,4 \pm 14,4$ & $1,6 \pm 1,6$ \\
\hline Isopoda & 0,00 & - & - & 0,07 & - & $1,6 \pm 1,6$ \\
\hline Dermaptera & 0,00 & - & - & 2,81 & $20,8 \pm 6,8$ & $43,2 \pm 12,9$ \\
\hline Protura & 0,00 & - & - & 6,33 & $136,0 \pm 19,7$ & $8,0 \pm 5,5$ \\
\hline Pauropoda & 0,20 & $3,2 \pm 3,2$ & $3,2 \pm 3,2$ & 1,27 & $22,4 \pm 11,0$ & $6,4 \pm 3,5$ \\
\hline Thysanoptera & 3,60 & $27,2 \pm 11,9$ & $89,6 \pm 20,5$ & 5,06 & $14,4 \pm 5,0$ & $100,8 \pm 25,5$ \\
\hline Araneae & 2,27 & $48,0 \pm 16,5$ & $25,6 \pm 9,3$ & 2,81 & $41,6 \pm 8,7$ & $22,4 \pm 8,0$ \\
\hline Chilopoda & 1,47 & $48,0 \pm 12,6$ & - & 0,77 & $12,6 \pm 5,2$ & $4,8 \pm 3,4$ \\
\hline Hymenoptera & 2,51 & $20,8 \pm 8,9$ & $60,8 \pm 14,9$ & 1,48 & $19,2 \pm 12,6$ & $14,4 \pm 5,0$ \\
\hline Pseudoscorpionida & 1,03 & $3,2 \pm 2,1$ & $30,4 \pm 12,7$ & 0,35 & $1,6 \pm 1,6$ & $6,4 \pm 4,3$ \\
\hline Coleoptera & 6,65 & $131,2 \pm 29,3$ & $84,8 \pm 13,1$ & 4,22 & $65,6 \pm 18,4$ & $30,4 \pm 13,4$ \\
\hline Collembola & 14,54 & $264,0 \pm 58,5$ & $208,0 \pm 72,6$ & 27,43 & $340,8 \pm 57,3$ & $283,2 \pm 65,8$ \\
\hline Larva de Coleoptera & 0,34 & $8,0 \pm 3,7$ & $3,2 \pm 3,2$ & 2,18 & $36,8 \pm 7,2$ & $12,8 \pm 5,2$ \\
\hline Larva de Diptera & 2,17 & $40,0 \pm 6,0$ & $30,4 \pm 17,4$ & 0,56 & $11,2 \pm 3,4$ & $1,6 \pm 1,6$ \\
\hline Larva de Lepidoptera & 1,48 & $19,2 \pm 7,6$ & $28,8 \pm 9,5$ & 1,27 & $8,0 \pm 2,7$ & $20,8 \pm 7,9$ \\
\hline Larva de Hymenoptera & 0,30 & $9,6 \pm 6,7$ & - & 0,00 & - & - \\
\hline Hemiptera & 0,54 & $16,0 \pm 5,8$ & $1,6 \pm 1,6$ & 0,28 & $4,8 \pm 2,4$ & $1,6 \pm 1,6$ \\
\hline Homoptera & 7,34 & $70,4 \pm 22,5$ & $168,0 \pm 50,0$ & 1,05 & $12,8 \pm 5,2$ & $11,2 \pm 5,4$ \\
\hline Diptera & 16,46 & $163,2 \pm 19,3$ & $371,2 \pm 52,6$ & 3,31 & $49,6 \pm 23,4$ & $25,6 \pm 6,8$ \\
\hline Lepidoptera & 0,15 & - & $4,8 \pm 3,4$ & 0,00 & - & - \\
\hline Trichoptera & 3,25 & $41,6 \pm 10,4$ & $64,0 \pm 15,8$ & 0,14 & - & - \\
\hline Scorpionida & 0,05 & - & $1,6 \pm 1,6$ & 0,00 & - & - \\
\hline Total de ind $/ \mathrm{m}^{2}$ & - & $1516,8 \pm 407,48$ & $1729,6 \pm 469,67$ & - & $1424 \pm 345,70$ & $851,2 \pm 273,95$ \\
\hline Riqueza de grupos & - & 21 & 21 & - & 23 & 22 \\
\hline
\end{tabular}

(1) ind $/ \mathrm{m}^{2} \pm$ e: abundância de indivíduos por metro quadrado \pm erro-padrão.

verão, bem como a maior ocorrência de chuvas nessa estação, concentrando $37 \%$ dos efetivos pluviométricos totais (Mattos, 2005), o que, segundo Moço et al. (2005), aumenta a oferta de alimentos para a fauna e cria ambiente mais favorável a essa. Paula et al. (2009) observam, em fragmentos florestais periodicamente inundados na Ilha da Marambaia, RJ, que as maiores taxas de deposição de serapilheira ocorrem durante a estação chuvosa.

No verão, Formicidae (28,44\%), Diptera (16,46\%), Collembola (14,54\%), Homoptera (7,34\%) e Coleoptera $(6,65 \%)$ foram os grupos de maior ocorrência, abrangendo 73,44\% dos efetivos totais da densidade de organismos da fauna edáfica, sendo os outros $26,56 \%$ representados pelos demais 16 grupos taxonômicos identificados.
Em um estudo conduzido em fragmentos florestais da restinga da Marambaia, RJ, na primavera, Souza et al. (2008) verificaram que os cinco grupos com maior participação na densidade total de invertebrados foram Formicidae (32,77 \%), larvas de Diptera (15,43\%), Coleoptera (10,64 \%), Diptera $(8,41 \%)$ e Collembola $(8,15 \%)$, o que corrobora com os dados observados neste estudo. É perceptível a importância dos insetos sociais da família Formicidae, não apenas por contribuírem expressivamente no total efetivo de artrópodos (Costa, 2002), mas por realizarem importantes funções no ambiente, como melhorar o arejamento e a infiltração da água no solo, fragmentar os detritos vegetais e incorporar a matéria orgânica ao solo (Souza et al., 2008; Silva et al., 2012a). 
Os grupos Coleoptera $(6,65 \%)$ e Collembola $(14,54 \%)$, apesar de menos expressivos, são também importantes organismos do sistema decompositor, podendo atuar tanto de forma direta quanto indireta. Segundo Moço et al. (2005), esses grupos de organismos podem comportar-se nos ecossistemas como saprófagos, fragmentando os resíduos vegetais, ou como predadores, alimentando-se de outros organismos; o grupo Collembola desenvolve a atividade predatória de microrganismos (Toledo, 2003; Tordoff et al., 2008), denominada microfagia, e possui também a fitofagia como hábito alimentar (Souza et al., 2008).

Quanto à expressividade de Diptera nos efetivos totais da densidade (16,46\%), acredita-se que esses utilizam o solo como refúgio, tendo sido coletados acidentalmente junto à serapilheira, uma vez que os ovos desses indivíduos podem ter eclodido durante coletas de solo, resultando nas larvas que foram coletadas.

No inverno, Formicidae e Collembola continuaram figurando entre os cinco grupos mais expressivos, predominando no sistema com 28,83 e $27,43 \%$, respectivamente, ao passo que o grupo Diptera representou apenas $3,31 \%$ e o Homoptera, $1,05 \%$ da frequência total de organismos. Contudo, acredita-se que essa relevante diminuição na frequência desses grupos taxonômicos não tem influência direta nos processos de decomposição do ecossistema, uma vez que são artrópodos classificados como não edáficos e sem funcionalidade conhecida nesse ambiente (Pinheiro, 1996; Moço et al., 2005).

O terceiro grupo de maior frequência relativa e, que não havia sido registrado na estação seca, foi o Protura, abrangendo 6,33\% do total. Segundo Costa (2002), os organismos da ordem Protura apresentam a saprofagia como hábito alimentar, sugerindo que na estação seca (inverno), esse grupo seja importante integrante do sistema decompositor, atuando diretamente na fragmentação da matéria orgânica.

Apesar de pouco expressivos, em ambas as estações, constatou-se a ocorrência dos grupos Symphyla e Pseudoscorpionida, assim como observado por Souza et al. (2008), em estudos conduzidos em fragmentos florestais também na restinga da Marambaia. Segundo esses autores, esses grupos são geralmente encontrados apenas em ambientes cujo ecossistema não apresenta degradação.

A relação entre a distribuição de indivíduos de cada grupo taxonômico e as épocas do ano em cada compartimento foi estudada por meio da análise de correspondência canônica (ACC) (Figura 1). As variáveis foram distribuídas em duas dimensões que explicaram $99,3 \%$ da variabilidade total entre as épocas do ano $(97,74 \%$, para a dimensão 1 e $2,16 \%$, para a 2). Ao longo da dimensão 1 (eixo principal), os compartimentos solo e serapilheira no verão estão agrupados na faixa à direita, enquanto no inverno estão dispostos na porção esquerda (Figura 1), o que evidencia o efeito das épocas do ano na distribuição dos grupos taxonômicos da fauna do solo.
Os grupos Collembola, Larva de Coleoptera, Protura e Dermaptera apresentaram maior associação com os compartimentos solo e serapilheira no inverno. Os grupos Formicidae, Coleoptera, Larva de Diptera e Aranae associaram-se ao compartimento solo no verão, enquanto Diplura e Hymenoptera estiveram mais relacionados à serapilheira no verão (Figura 1).

A ACC indicou que os grupos da fauna edáfica são responsivos às variações ambientais existentes entre as estações. A maior ou menor associação de determinado grupo da fauna edáfica, em cada época, deve-se, possivelmente, às condições de umidade (intermitência de inundação) e temperatura do soloserapilheira nesse ambiente.

Em razão das características relacionadas ao uso do habitat e à principal forma de utilização do recurso alimentar (Costa, 2002), os grupos taxonômicos identificados foram reunidos em sete grupos funcionais (Costa, 2002; Silva et al., 2012b) (Quadro 2), sendo classificado como grupo "Outros" aqueles organismos sem funcionalidade conhecida. Segundo Correia \& Oliveira (2000), a análise dos grupos funcionais nos ecossistemas tem como uma de suas limitações a impossibilidade de determinar-se a funcionalidade exata de vários dos grupos da fauna do solo, em nível de classe, ordem ou até mesmo família. Contudo, neste estudo, essa limitação pôde ser contornada, uma vez

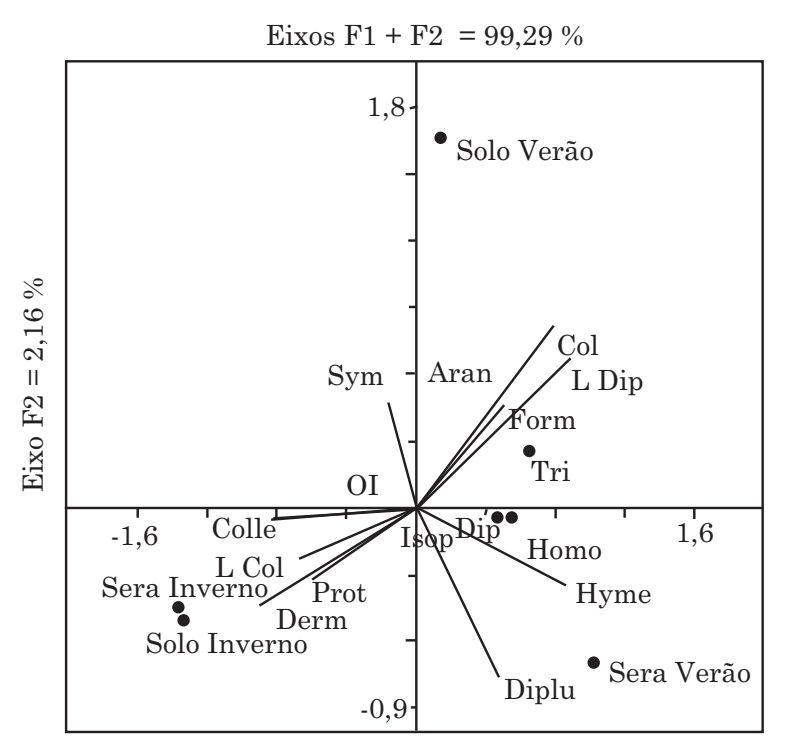

Eixo F1 = 97,74 \%

Figura 1. Análise de correspondência canônica para abundância de grupos da fauna do solo nos compartimentos solo e serapilheira (Sera), nas estações verão e inverno na restinga da Marambaia, RJ. OI: outros invertebrados; Diplu: Diplura; Dip: Diptera; Colle: Collembola; L: Larva; Derm: Dermaptera; Isop: Isoptera; Homo: Homoptera; Hyme: Hymenoptera; Symphyla; Aran: Aranae; Col: Coleoptera; Form: Formicidae; Tri: Trichoptera. 
que se trabalhou com a comparação entre diferentes épocas do ano dentro de uma mesma comunidade, dando-se enfoque às modificações impostas por componentes ambientais.

O grupo funcional de maior abrangência, para as duas estações estudadas (verão e inverno), foi o dos insetos Sociais, Formicidae e Isoptera (Quadro 2), ratificando a importância desse no sistema decompositor em ecossistemas de restinga, seja de forma direta ou indireta, uma vez que, junto com o grupo funcional Larvas (menos expressivos), podem apresentar hábitos alimentares tanto de saprofagia quanto de predação (Moço et al., 2005). Além disso, os insetos Sociais atuam efetivamente na agregação do solo (Pinheiro, 1996; Oliveira et al., 2012) pela escavação e construção de ninhos (Oliveira et al., 2012) e, dessa forma, se fazem importantes elementos do ecossistema estudado.

Outro grupo que também esteve presente de forma expressiva, em ambas as estações, foram os Micrófagos (Quadro 2), representados pelos Collembola, que desenvolvem a atividade predatória de microrganismos (Tordoff et al., 2008). Além disso, influenciam indiretamente na fertilidade do solo, produzem enzimas e fragmentam a matéria orgânica (Berg et al., 2004; Yang et al., 2012).

No inverno, os Saprófagos $(22,57 \%)$ aparecem como o terceiro grupo de maior abrangência, seguido dos Predadores (9,63\%). Padrão inverso foi observado na estação de verão, quando os Predadores representaram 13,95\% dos grupos funcionais e os Saprófagos, 9,27 \% (Quadro 2), sugerindo que o sistema decompositor é menos ativo nessa estação. Contudo, se por um lado a atividade decompositora pode estar reduzida no inverno, a maior atividade dos predadores nessa estação, é fundamental para o ecossistema, uma vez que os predadores, como os representantes do grupo Aranae, são importantes organismos na manutenção do equilíbrio ecológico da fauna edáfica (Toledo, 2003). Os grupos Fitófagos e Outros, que juntos somam
27,8 \%, no verão, e 4,79 \%, no inverno (Quadro 2), são representados por artrópodos classificados como não edáficos e sem funcionalidade definida (Pinheiro, 1996; Moço et al., 2005), não participando, então, do sistema decompositor.

Dessa forma, de maneira geral, a microfagia e saprofagia representaram as principais formas de utilização dos recursos alimentares, seguidas da predação, padrão esse semelhante ao observado por Costa (2002), em plantios de leguminosas arbóreas em Seropédica, RJ.

Com relação à distribuição dos organismos (grupos funcionais) nos compartimentos solo e serapilheira, observou-se a existência de distribuição irregular entre essas camadas para as duas estações estudadas (Figura 2). No entanto, quando se avaliou a fauna do solo de maneira geral (Fauna do solo total - FT), verificou-se, no verão, a preferência dos organismos pelo compartimento serapilheira (54\%), em detrimento do solo $(46 \%)\left(\chi^{2}=459,9 ; p=0,01\right)$ (Figura 2). Esse padrão pode ser por causa da maior ocorrência de chuvas nessa estação, o que, segundo Moço et al. (2005), além de estimular o crescimento vegetal e, consequentemente, a produção de serapilheira, aumenta a oferta de alimentos para a fauna, cria ambiente favorável para a biota do solo e estimula a atividade biológica.

Em estudos realizados na restinga em Maricá, RJ, representada por uma vegetação de distribuição espacial gregária e de porte reduzido, Oliveira (1997) verificou a preferência da fauna edáfica pelo compartimento solo, em detrimento da serapilheira, apontando como principais causas dessa preferência a qualidade do recurso alimentar e as temperaturas mais elevadas das camadas superiores da serapilheira.

Contudo, diferindo da restinga estudada por Oliveira (1997), a restinga da Marambaia apresenta fitofisionomia arbórea de elevado porte (Mattos, 2005), que promove sombreamento completo da área, amenizando, dessa forma, os gradientes de

Quadro 2. Distribuição percentual dos grupos taxonômicos em grupos funcionais, em razão das características relacionadas ao uso do habitat e à principal forma de utilização do recurso alimentar, para as duas estações estudadas (verão e inverno)

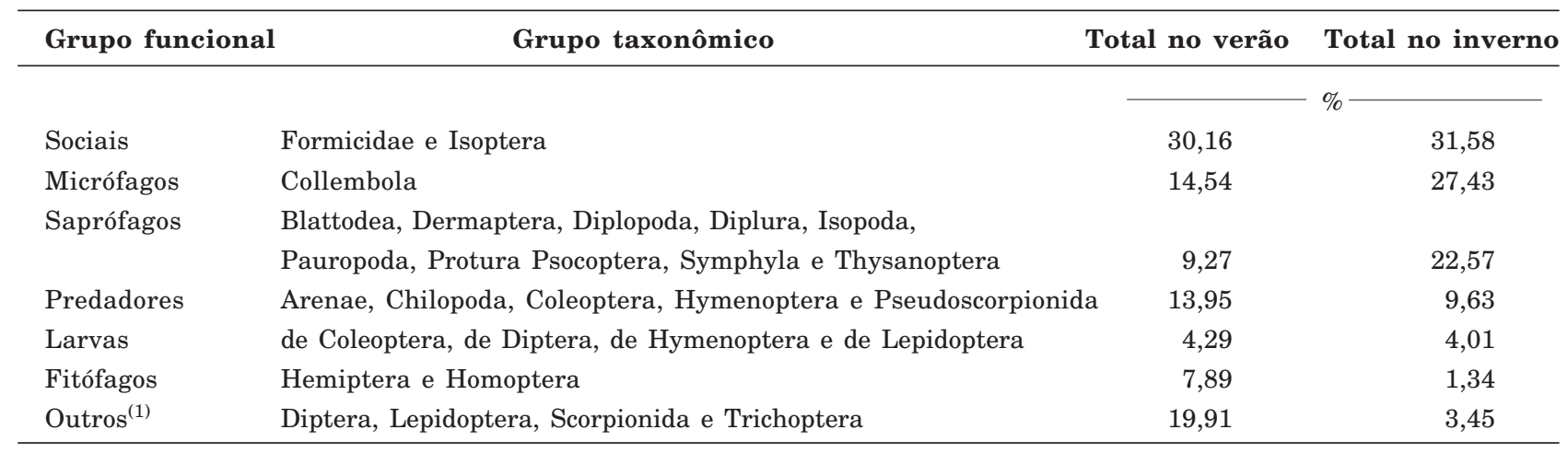

(1) Sem funcionalidade conhecida. 

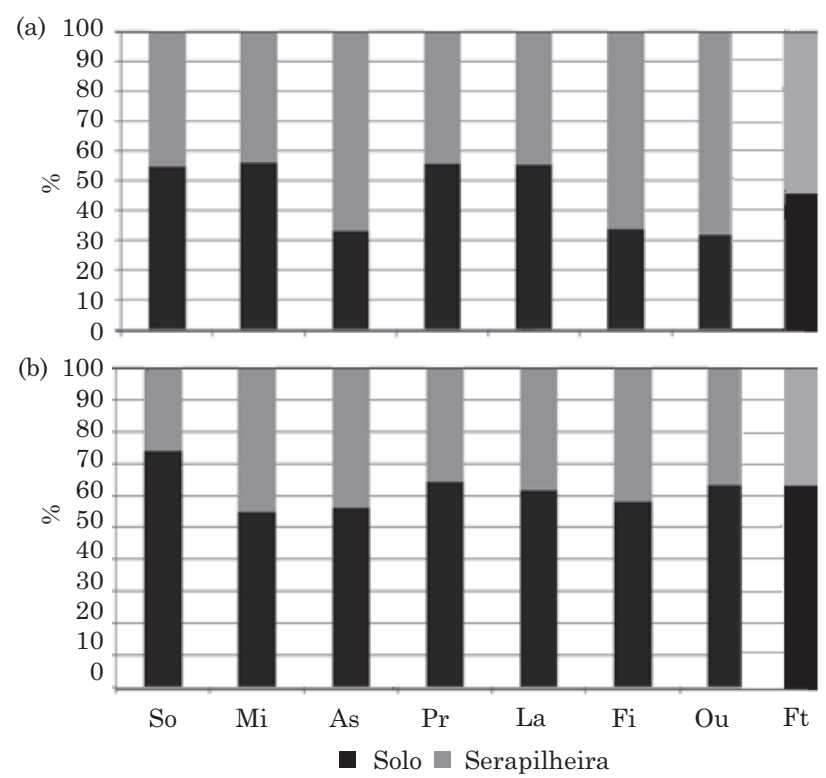

Figura 2. Distribuição vertical da fauna do solo total (FT) e dos grupos funcionais nos compartimentos solo e serapilheira para as duas estações estudadas, verão (a) e inverno (b). So: Insetos sociais; Mi: Micrófagos; As: Saprófagos; Pr: Predadores; La: Larvas; Fi: Fitófagos; e Ou: Outros.

temperatura e os efeitos nocivos da radiação solar excessiva, verificado em restingas abertas de moitas.

Sugere-se, então, que o principal fator que induz a migração da fauna edáfica entre os compartimentos do solo da área avaliada neste estudo é a pluviosidade, que gera o afloramento do lençol freático. Esse, por se encontrar muito próximo à superfície, promove o alagamento parcial do ambiente na estação chuvosa, fazendo com que a fauna migre para a serapilheira pelas condições mais favoráveis no verão. Esse evento contribui também para alimentação, uma vez que o maior grau de hidromorfismo, ao retardar a velocidade de decomposição da matéria orgânica, promove o seu acúmulo (Souza et al., 2008), podendo proporcionar maior disponibilidade de alimento para os organismos associados e consequente migração do solo para serapilheira.

No inverno, verificou-se maior abundância de indivíduos no solo $(63 \%)$ em relação à serapilheira $(37 \%)\left(\chi^{2}=417,31 ; \mathrm{p}=0,01\right)$ (Figura 2$)$, o que é corroborado por Toledo (2003). A estação do inverno da área estudada caracteriza-se por apresentar baixos índices pluviométricos, menores valores de umidade relativa, menores graus de nebulosidade e maior insolação que o verão (Mattos, 2005), o que torna essa estação muito seca. Dessa forma, alguns grupos de invertebrados tendem a migrar das camadas superficiais, que apresentam maiores conteúdos de matéria orgânica do solo, quando essas evidenciam baixos conteúdos de água, para a camada mineral subsuperficial (Bandeira \& Harada, 1998). Esse comportamento representa possivelmente estratégia da fauna edáfica em buscar refúgio nas camadas mais internas, visando suportar períodos desfavoráveis à sua sobrevivência (Oliveira, 1997).

\section{CONCLUSÕES}

1. O grupo de maior ocorrência, em ambas as estações, foi Formicidae, seguido de Collembola, ratificando a importância desses grupos para a fragmentação dos detritos vegetais em ecossistemas de restinga. Não foram verificadas diferenças na riqueza dos grupos taxonômicos da fauna do solo em razão da sazonalidade na restinga da Marambaia.

2. A abundância da fauna aumentou do inverno para o verão, indicando que a precipitação pluvial, que promove afloramento do lençol freático, induz a migração da fauna entre os compartimentos do solo.

3. Os organismos procuram mais a serapilheira, no verão, e o solo, no inverno.

4. A ocorrência de grupos taxonômicos da fauna edáfica, que só são percebidos em ambientes não perturbados, indica que o ecossistema de restinga da Ilha da Marambaia apresentava baixo grau de alteração antrópica, na época em que foi realizado este estudo.

\section{LITERATURA CITADA}

BANDEIRA, A.G. \& HARADA, A.Y. Densidade e distribuição vertical de macroinvertebrados em solos Argilosos e Arenosos na Amazônia Central. Acta Amaz., 28:191-204, 1998.

BERG, M.P.; STOFFER, M. \& van DEN HEUVEL, H.H. Feeding guilds in Collembola based on digestive enzymes. Pedobiologia, 48:589-601, 2004.

BIGNELL, D.E. Termites as soil engineers and soil processors. In: KONIG, H. \& VARMA, A., eds. Soil biology. Intestinal microorganisms of soil invertebrates. 6.ed. Berlin, Springer-Verlag, 2006. p.183-220.

BRIAR, S.S.; FONTE, S.J.; PARK, I.; SIX, J.; SCOWC, K. \& FERRIS, H. The distribution of nematodes and soil microbial communities across soil aggregate fractions and farm management systems. Soil Biol. Biochem., 43:905914, 2011.

CARRILLO,Y.; BALL, B.A.; BRADFORD, M.A.; JORDAN, C.F. \& MOLINA, M. Soil fauna alter the effects of litter composition on nitrogen cycling in a mineral soil. Soil Biol. Biochem., 43:1440-1449, 2011.

CORRÊA NETO, T.A.; PEREIRA, M.G.; CORREIA, M.F. \& ANJOS, L.C. Deposição de serapilheira e fauna edáfica em áreas de Eucalipto e Floresta Secundária. Flor. Amb., 8:70-75, 2001. 
CORREIA, M.E.F. Organização da Comunidade de Macroartrópodos Edáficos em um Ecossistema de Mata Atlântica de Tabuleiros, Linhares (E.S.). Rio de Janeiro, Universidade Federal do Rio de Janeiro, 1994. 78p. (Dissertação de Mestrado)

CORREIA, M.E.F. \& OLIVEIRA, L.C.M. Fauna de solo: Aspectos gerais e metodológicos. Seropédica, Embrapa Centro Nacional de Pesquisa de Agrobiologia, 2000. 46p. (Documento, 112)

COSTA, P. Fauna do solo em plantios experimentais de Eucalyptus grandis Maiden, Pseudosamanea guachapele Dugand e Acacia mangium Wild. Seropédica, Universidade Federal Rural do Rio de Janeiro, 2002. 93p. (Dissertação de Mestrado)

DUPONT, S.T.; FERRIS, H \& van HORN, M. Effects of cover crop quality and quantity on nematode-based soil food webs and nutrient cycling. Appl. Soil Ecol., 41:157-167, 2009.

EGGLETON, P.; INWARD, K.; SMITH, J.; JONES, D. \& SHERLOCK, E. A six year study of earthworm (Lumbricidae) populations in pasture woodland in southern England shows their responses to soil temperature and soil moisture. Soil Biol. Biochem., 41:1857-1865, 2009.

FERNANDES, M.M.; MAGALHÃES, L.M.S.; PEREIRA, M.G.; CORREIA, M.E.F.; BRITO, R.J. \& MOURA, M.R. Influência de diferentes coberturas florestais na fauna do solo na Flona Mário Xavier, no município de Seropédica, RJ. Floresta, 41:533-540, 2011.

GARAY, I. Relations entre l'hetérogéinéité des litières et l'organization des peuplements d'arthropodes édaphiques. Paris, École Normale Supérieure, 1989. 192p. (Publications du Laboratoire de Zoologie n.35)

GIRACCA, E.M.N.; ANTONIOLLE, Z.I.; ELTZ, F.L.F.; BENEDETTI, E.; LASTA, E.; VENTURINI, S.F.; EVANDRO, F. \& BENEDETTI, T. Levantamento da meso e macrofauna do solo na Microbacia do Arroio Lino, Agudo/ RS. R. Bras. Agroci., 9:257-26, 2003.

GOMES, J.B.V.; BARRETO, A.C.; MICHEREFF FILHO, M.; VIDAL, W.C.L.; COSTA, J.L.S.; OLIVEIRA-FILHO, A.T. \& CURI, N. Relações entre atributos do solo e atividade de formigas em restingas. R. Bras. Ci. Solo, 34:67-78, 2010.

HAMMER, O.; HARPER, D.A. \& RYAN, P.D. PAST Paleontological Statistics ver.1.12 2004. Disponível em: $<$ http://www.folk.uio.no/ohammer/past>. Acesso em: 13 out. 2012 .

HOLMSTRUP, M.; SORENSEN, J.G.; MARALDO, K.; SCHMIDT, I.K.; MASON, S.; TIETEMAD, A.; SMITH, A.R.; EMMETT, B.; SCHMELZ, R.M.; BATAILLON, T.; BEIER, C. \& EHLERS, B.K. Increased frequency of drought reduces species richness of enchytraeid communities in both wet and dry heathland soils. Soil Biol. Biochem., $53: 43-49,2012$

KÖPPEN, W. Climatologia: con un estudio de los climas de la tierra. México, Fondo de Cultura Econômica, 1948. 479p.

LAVELLE, P.; BLANCHART, E.; MARTIN, A.; MARTIN, S.; SPAIN, A.; TOUTAIN, F.; BAROIS, I. \& SCHAEFER, R. A hierarchical model for decomposition in terrestrial ecosystems: application to soils of the humid tropics. Biotropica, 25:130-150, 1993.
MANLAY, R.J.; CADET, P.; THIOULOUSE, J. \& CHOTTE, J. Relationships between abiotic and biotic soil properties during fallow periods in the sudanian zone of Senegal. Appl. Soil Ecol., 14:89-101, 2000.

MATTOS, C.C.L.V. Caracterização climática da Restinga da Marambaia. In: MENEZES, L.F.T.; ARAUJO, D.S. \& PEIXOTO, A.L., eds. História Natural da Marambaia. Rio de Janeiro, Editora Universidade Rural, 2005. p.5566.

MENEZES, L.T.; PEIXOTO, A.L. \& ARAÚJO, D.S.D. História Natural da Marambaia. Seropédica, EDUR, 2005. 288p.

MOÇO, M.K.S.; GAMA-RODRIGUES, E.F.; GAMARODRIGUES, A.C. \& CORREIA, M.E.F. Caracterização da Fauna Edáfica em diferentes coberturas vegetais na região Norte Fluminense. R. Bras. Ci. Solo, 29:555-564, 2005 .

ODUM, E.P. Ecologia. Ed. Guanabara Koogan, Rio de Janeiro, 1988. 343p.

OLIVEIRA, L.C.M. Caracterização da comunidade de macroartrópodos edáficos em uma Mata de Restinga, Maricá (RJ). Rio de Janeiro, Universidade Federal do Rio de Janeiro, 1997. 92p. (Dissertação de Mestrado)

OLIVEIRA, M.I.L.; BENITO, N.P.; CAMARGO, A.J.A.; GUIMARÃES, M.F. \& BROSSARD, M. Atividade de colônias de Cornitermes cumulans (Isoptera, Nasutitermitinae) sobre estruturas edáficas macro e microagregadas em casa de vegetação. Semina Ci. Agrar., 33:1733-1744, 2012.

OSLER, G.H.R. \& SOMMERKORN, M. Toward a complete soil $\mathrm{C}$ and $\mathrm{N}$ cycle: Incorporating the soil fauna. Ecology, 88:1611-1621, 2007.

OYEDELE, D.J.; SCHJENNING, P. \& AMUSAN, A.A. Physicochemical properties of earthworm casts and uningested parent soil from selected sites in southwestern Nigeria. Ecol. Eng., 28:106-113, 2006.

PAULA, R.R. Caracterização edáfica e ciclagem de nutrientes em complexo florestal periodicamente inundável na Ilha da Marambaia-RJ. Seropédica, Universidade Federal Rural do Rio de Janeiro, 2009. 47p. (Monografia do Curso de Engenharia Florestal)

PAULA, R.R.; PEREIRA, M.G. \& MENEZES, L.F.T. Aporte de nutrientes e decomposição da serapilheira em três fragmentos florestais periodicamente inundados na ilha da Marambaia, RJ. Ci. Flor., 19:139-148, 2009.

PEREIRA, M.G.; SILVA, A.N.; PAULA, R.R. \& MENEZES, L.F.T. Aporte e decomposição de serapilheira em floresta periodicamente inundável na restinga da Marambaia, RJ. Ci. Flor., 22:59-67, 2012.

PIMENTEL-GOMES, F. Curso de estatística experimental. 14.ed. Piracicaba: Degaspari, 2000. 477p.

PINHEIRO, L.B.A. Estudo da macrofauna de solos cultivados com cana de açúcar sob diferentes manejos de colheita crua e queimada. Seropédica, Universidade Federal Rural do Rio de Janeiro, 1996. 100p. (Dissertação de Mestrado) 
SCHEU, S. Effects of earthworms on plant growth: Patterns and perspectives. Pedobiologia 47:846-856, 2003.

SIDDIKY, M.D.; REZAUL, K.; KOHLER, J.; COSME, M. \& RILLIG, M.C. Soil biota effects on soil structure: Interactions between arbuscular mycorrizal fungal mycelium and collembolan (Repost). Soil Biol. Biochem., 50:33-39, 2012.

SILVA, J.; JUCKSCH, I. \& TAVARES, R.C. Invertebrados edáficos em diferentes sistemas de manejo do cafeeiro na Zona da Mata de Minas Gerais. R. Bras. Agroecol., 7:112125, 2012a.

SILVA, J.; JUCKSCH, I.; FERES, C.I.M.A. \& TAVARES, R.C. Fauna do solo em sistemas de manejo com café. J. Biotechnol. Biodiver., 3:59-71, 2012b.

SILVA, R.F. Roça Caiçara: Dinâmica de nutrientes, propriedades físicas e fauna do solo em um ciclo de cultura. Seropédica, Universidade Federal Rural do Rio de Janeiro, 1998. 165p. (Dissertação de Mestrado)

SIMPSON, J.E.; SLADE, E.; RIUTTA, T. \& TAYLOR, M.E. Factors affecting soil fauna feeding activity in a fragmented lowland temperate deciduous woodland. PLoS ONE, 7:1-6, 2012.
SOARES, M.I.J. \& COSTA, E.C. Fauna do solo em áreas com Eucalyptus spp. e Pinus elliottii, Santa Maria, RS. Ci. Flor., 11:29-43, 2001.

SOUZA, R.C.; CORREIA, M.E.F.; PEREIRA, M.G.; SILVA, E.M.R.; PAULA, R.R. \& MENEZES, L.F.T. Estrutura da comunidade da fauna edáfica em fragmentos florestais na Restinga da Marambaia, RJ. R. Bras. Ci. Agrár., 3:49$57,2008$.

TOLEDO, L. O. Aporte de serapilheira, fauna edáfica e taxa de decomposição em áreas de floresta secundária no município de Pinheiral, RJ. Seropédica, Universidade Federal Rural do Rio de Janeiro, 2003. 80p. (Dissertação de Mestrado)

TORDOFF, G.M.; BODDY, L. \& JONES, T.H. Species-specific impacts of Collembola grazing on fungal foraging ecology. Soil Biol. Biochem., 40:434-442, 2008.

YANG, X.; YANG, Z.; WARREN, M.W. \& CHEN, J. Mechanical fragmentation enhances the contribution of Collembola to leaf litter decomposition. Eur. J. Soil Biol., 53:23-31, 2012.

YANG, X. \& CHEN, J. Plant litter quality influences the contribution of soil fauna to litter decomposition in humid tropical forests, southwestern China. Soil Biol. Biochem., 41:910-918, 2009. 